\title{
Deploying Wireless Sensors for Differentiated Coverage and Probabilistic Connectivity
}

\author{
Yanjun $\mathrm{Li}^{\dagger \ddagger}$, Ye-Qiong Song ${ }^{\ddagger}$, Yi-hua Zhu ${ }^{\dagger}$, René Schott ${ }^{\ddagger}$, \\ ${ }^{\dagger}$ College of Computer Science and Technology, Zhejiang University of Technology, Hangzhou, China \\ ${ }_{\ddagger}^{\ddagger}$ LORIA-Nancy University, Vandoeuvre-lès-Nancy, France \\ Email: yjli.iipc@gmail.com, song@loria.fr, yhzhu@zjut.edu.cn, schott@loria.fr
}

\begin{abstract}
The deployment strategy for achieving differentiated coverage and probabilistic connectivity in wireless sensor networks is studied in this paper. A novel solution based on elitist non-dominated sorting genetic algorithm (NSGA-II) is proposed. Simulation results show that NSGA-II based strategy can meet the desired coverage requirements and maintain connectivity in a probabilistic manner with a relatively small number of sensors. In addition, for the applications in which the coverage requirement varies in some subareas, a local genetic operation is more timeefficient and needs less variation in the original disposal than a renewed global optimization.
\end{abstract}

\section{INTRODUCTION}

Coverage and connectivity are two fundamental issues in wireless sensor networks. The goal of coverage is to ensure that the area of interest (AoI) or the physical state of the target is precisely sensed, while connectivity is to ensure the event in AoI or the state of the target is successfully transmitted to the base station and subsequently captured by remote users. Coverage and connectivity together can be treated as a measure of quality of service (QoS). In the case where the target field is inaccessible, random deployment is the only method. However, it cannot guarantee the required coverage and connectivity unless the node density is higher than certain threshold [1]. If the target field is accessible, a deterministic sensor deployment strategy should be more effective. Such a strategy would minimize the total number of sensors required and achieve the specific needs of applications in terms of their expected quality of coverage and connectivity.

Most existing works on sensor deployment with guaranteed coverage and connectivity are based on simplistic sensing and communication models, such as the boolean disc model [1-3], with sensing range $R_{s}$ and communication range $R_{c}$. Under disc model, it is well known results that when $R_{c} \geq 2 R_{s}$, $k$-coverage of a convex region implies $k$-connectivity [2]. However, this result may not hold under probabilistic sensing and communication models. Although disk model facilitates a geometric treatment to the coverage and connectivity problem, it fails to consider collaboration in sensing and stochastic nature of radio communication. Our work is based on a probabilistic sensing model and a shadowing fading link model, which are more realistic compared to boolean disk model.

In this paper, we take into consideration the geographic feature of the AoI and the target event. In other words, different points of interest (PoIs) in the AoI can be of different importance due to their geographic relation to the events. If there is some a priori knowledge about the importance of each PoI, the deployment plan can be more customized. For example, in case of a fire detection application, PoIs around the oil depot are considered highly risky and should be assigned high coverage probability, while the PoIs near the water area can be placed lower importance. Intuitively, locations with higher detection requirement need to be deployed more sensors than those with lower requirement. Meanwhile, the network connectivity should be guaranteed in a probabilistic manner otherwise more power has to be used to ensure communication. This deployment issue can be formulated as a multi-objective optimization problem (MOP). The essential requirement for MOP is to find Pareto-optimal solutions under multiple decision objectives and constraints, from which the user can make a trade-off choice according to their own preference and some practical limits. Among the existing solutions, genetic algorithm (GA) has been recognized as one of the possibly well-suited to MOP [4]. We adopt NSGAII [4], which is one of the most popular GAs and has been shown effective in a number of applications [5, 6], to develop the framework for solving the MOP in sensor deployment. The algorithm can be run off-line a priori and the sensor nodes are distributed following the final result. In case the user changes a small part of the detection requirement, it is not efficient to run the algorithm once again over the whole area. We propose to restrict genetic operations in the varied subareas while evaluate the fitness function globally. Simulation shows that this method converges more quickly with little degradation in performance and the variation of sensor nodes are much less than the global way.

The rest of the paper is organized as follows. Section II reviews related work. Section III introduces the models used and problem definition. In Section IV, NSGA-II based deployment strategy is specified and analyzed. We present simulation results in Section $\mathrm{V}$ and conclude the paper in Section VI.

\section{RELATED WORK}

The problem of finding optimal deployment with guaranteed coverage and connectivity has been addressed in several literatures. Bai et al. [3] propose optimal deployment patterns to achieve full coverage and $k$-connectivity $(k \leq 6)$ under different ratios of sensor communication range over sensing range. However, their work is under the assumption of disk 
sensing and communication model. Under non-disc models, their "optimal" deployment patterns may be too conservative. A few literatures have replaced disk sensing model by probabilistic [7-9] or stochastic models. Zou et al. [7] present two heuristic algorithms to optimize the number of sensors while minimizing the overall miss probability and they specifically deal with the problem of uncertainty in sensor locations. In [8], Zhang et al. formulate the differentiated deployment problem as an integer linear programming problem and also propose a heuristic algorithm to solve it. Propositions in $[7,8]$ only satisfy coverage requirement but do not consider network connectivity. Aitsaadi et al. [9] use tabu search to find optimal deployment with differentiated coverage and connectivity. Their approach is testified to outperform solutions in $[7,8]$. However, they adopt disk model as the communication model and their final solution has no diversity. There are also literatures addressing mobile sensor deployment [10], while in this paper, we only focus on static sensor node deployment, i.e., sensors do not move after deployed.

\section{Preliminaries ANd Problem Formulation}

\section{A. Sensing and Communication Models}

We adopt exponential sensing model as described in [7]. Specifically, the probability that a target/event at point $P$ be sensed by sensor $s_{i}$ is:

$$
c_{s_{i}}(P)= \begin{cases}1, & h\left(s_{i}, P\right) \leq r_{s} \\ e^{-\omega\left(h\left(s_{i}, P\right)-r_{s}\right)^{\beta}}, & r_{s}<h\left(s_{i}, P\right)<r_{u} \\ 0, & h\left(s_{i}, P\right) \geq r_{u} .\end{cases}
$$

where $h\left(s_{i}, P\right)$ is the distance between $s_{i}$ and $P, \omega$ and $\beta$ are parameters that measure the decay of sensing probability with distance, $r_{s}$ and $r_{u}$ are two thresholds denoting boolean sensing radius and maximum sensing range respectively. This model reflects the behavior of range sensing devices such as infrared and ultra sound sensors.

Let $\mathcal{S}$ be the set of nodes whose sensing range cover the point $P$, expressible as $\mathcal{S}=\left\{s_{i} \mid h\left(s_{i}, P\right)<r_{u}\right\}$. The total coverage probability of the point $P$, also referred to as 1coverage probability, is defined as:

$$
C_{\mathcal{S}}(P)=1-\prod_{i=1}^{k}\left(1-c_{s_{i}}(P)\right)
$$

which is the probability that point $P$ is covered by at least one sensor.

Empirical studies have shown that the radio links between low-power sensing devices are extremely unreliable. Thus boolean disk model has been repeatedly challenged. Here we adopt $P R R$ (packet reception rate)-based link model [11], which can reflect the unreliable and asymmetric nature of wireless links. The $P R R$ of a wireless link is expressible as:

$$
p(h)=\left(1-\frac{1}{2} \exp \left(-\frac{\gamma(h)}{2} \frac{1}{0.64}\right)\right)^{8 f}
$$

where $h$ is the transmitter-receiver distance, $\gamma(h)$ is the signalto-noise ratio (SNR), and $f$ is the frame size including preamble, payload and CRC in following MICA2 motes [12] and the standard non-coherent FSK modulation and NRZ coding. This model takes into account both distance-dependent path loss and log-normal shadowing in identifying the signal strength and randomness in wireless environment.

For transmitting power $P_{t}$, the $\mathrm{SNR}, \gamma(h)$, is expressible as:

$$
\gamma(h)_{d B}=P_{t d B}-P L(h)_{d B}-P_{n d B}
$$

where, by MICA2 radios, $P_{t d B}$ is set at $0 \mathrm{~dB}$, the noise floor $P_{n d B}$ is at $-115 \mathrm{~dB}$, while the path loss $P L(h)_{d B}$ is modeled as:

$$
P L(h)_{d B}=P L\left(h_{0}\right)_{d B}-10 n \log _{10}\left(h / h_{0}\right)+X_{\sigma d B}
$$

in which $n$ is the path loss exponent, $h_{0}$ is the reference distance ( 1 meter), and $X_{\sigma}$ denotes the log-normal shadowing with zero mean and variance $\sigma^{2}$.

\section{B. Problem Formulation}

The to-be-deployed area $\mathcal{A}$ is first discretized into $X \times Y$ grids. The grid dimension depends on the user's precision requirement and computational capability. If the area is highly fine-grained, each grid can be regarded as a PoI. To facilitate mathematical description, we refer to each grid by its barycenter. The distance between two grids is defined as the distance between their barycenters. As mentioned before, the coverage requirement of different $\mathrm{PoI}$ in the area $\mathcal{A}$ is different characterized by their geographic relation to events or targets. Thus, we assume that each PoI $P_{i}$ in $\mathcal{A}$ is associated with a minimum coverage threshold, denoted $R_{i}, 0 \leq R_{i} \leq 1$. The deployment strategy can thus be denoted by a $X \times Y$ boolean matrix $D$, with the element $d_{i}=1$ indicating a deployed sensor in respective PoI while $d_{i}=0$ no sensor deployed. After deployment, each PoI is actually covered with probability $C_{i}$, defined by (2). We use $G(V, E)$ to represent the communication graph after deployment, in which $V$ is the vertex set and $E$ is the edge set denoting communication links. Finally, under given connectivity requirement $R_{\text {conn }}$, we formulate the MOP as:

$$
\begin{aligned}
& \min \quad N=\sum_{i=1}^{X \times Y} d_{i}, \\
& \min \sum_{i=1}^{X \times Y} \Delta_{i}, \\
& \text { s.t. } \operatorname{Conn}(G(V, E)) \geq R_{\text {conn }}, \\
& d_{i} \in\{0,1\} .
\end{aligned}
$$

where

$$
\Delta_{i}= \begin{cases}\left(R_{i}-C_{i}\right) / R_{i}, & R_{i}>C_{i} \\ 0, & R_{i} \leq C_{i} .\end{cases}
$$

and Conn is defined as the 1-connectivity probability of the resulting network. In problem (6), the first objective is to minimize the number of nodes while the second objective is to minimize the difference between the required coverage threshold and the after-deployment coverage probability. The constraint is that the 1-connectivity probability of the resulting network should be greater than user requirement $R_{\text {conn } n}$. The 
dimension of the solution space of the above problem is $2^{X \times Y}$. To obtain the optimal or sub-optimal solutions in polynomial time, we resort to heuristic approaches.

\section{NSGA-II BASED DEPLOYMENT STRATEGY}

There are a number of heuristic methods possible to solve problem (6), among which we choose a popular genetic algorithm, NSGA-II. As well-known, GAs have been successfully applied in many areas, especially in numerical and combinatorial optimizations [5, 6]. NSGA-II follows some basic steps of GA. The population is first initialized and then sorted based on nondomination into each front by using a fast sorting algorithm. Each individual in each front is assigned a fitness (or rank) value equal to its nondomination level. Once the nondominated sorting is completed the crowding distance is also assigned. Parents are selected from the population by using binary tournament selection based on the rank and crowding distance. The offspring population is combined with the current generation population and selection is performed to set the individuals of the next generation. The selected parents generate offspring by using crossover and mutation operators. The new generation is controlled by each front subsequently until the population size exceeds the current population size. Since all the previous and current best individuals are added in the combined population, elitism is ensured in the NSGA-II.

\section{A. Initial Population}

Each GA requires an initial population $P_{0}$ to serve as the starting point. To have more diversity in the initial population, we use both random and greedy approaches to generate $P_{0}$. Random deployment is calculated as follows:

$$
d_{i}= \begin{cases}1, & \text { rand }<K \cdot R_{i} \\ 0, & \text { otherwise }\end{cases}
$$

in which $K$ is a tunable parameter controlling the node density and rand $\in[0,1]$. The greedy approach is similar to the initialization procedure presented in [9]. The decision to deploy a sensor on $P_{i}$ follows Bernoulli distribution $\mathcal{B}(\theta)$, whose parameter $\theta$ associated to $P_{i}$ is calculated as follows:

$$
\theta=\frac{1}{\left|\mathcal{S}\left(P_{i}\right)\right|} \sum_{i=1}^{\left|\mathcal{S}\left(P_{i}\right)\right|} 1_{\left\{C_{i}<R_{i}\right\}}
$$

where $\left|\mathcal{S}\left(P_{i}\right)\right|$ is the number of nodes in $\mathcal{S}$, and $1_{\{\cdot\}}$ is the indicating function, which is equal to 1 if the condition is true and 0 otherwise. First, there is no node deployed. For any PoI $P_{i}, C_{i}=0$. Select a random PoI and compute the value of $\theta$ following (9). Second, generate a list $L$ to include all points of $\mathcal{A}$ in decreasing order of the value of $\theta$. Third, select the point $P_{i}$ with highest $\theta$ and remove it from $L$, and compare the actual sensing probability $C_{i}$ with the requirement $R_{i}$. If $C_{i}<R_{i}$, deploy a sensor in $P_{i}$ following $\mathcal{B}(\theta)$. If the decision is to deploy a sensor, recompute the coverage probability in the sensing range of $P_{i}$ and update $L$. Repeat the third step until the list $L$ is empty.

\section{B. Objective Evaluation, Fast Sorting and Crowding Distance Comparison}

After the initial population is generated, we compute all the objectives for each individual solution and evaluate the constraints, following equation 10 .

$$
\begin{aligned}
& F_{1}=\sum_{i=1}^{X \times Y} d_{i}, \quad F_{2}=\sum_{i=1}^{X \times Y} \Delta_{i}, \\
& C t=\operatorname{Conn}(G) .
\end{aligned}
$$

After computing the objectives and constraints, each individual solution in the population is ranked with nondominated criteria and sorted into different fronts by using a fast sorting algorithm. Crowding distance is computed for differentiating the solutions of the same rank. Thus the diversity among nondominated solutions can be obtained.

\section{Genetic Operations}

An intermediate population of size $n_{p}$ is created by employing the following genetic operators:

1) Selection: First, parents are selected from the population by using a binary tournament selection based on the rank and crowding distance. This is based on the principle that parents with better chromosomes can reproduce better offsprings.

2) Crossover: We choose multiple crossover points, whose locations are calculated using a random number generator (RNG), to create a new population with probability $p_{c}$.

3) Mutation: Newly reproduced chromosomes are transferred to the mutation pool. With mutation probability $p_{m}$, randomly chosen chromosomes are mutated. A new population is thus generated. To achieve good convergence, $p_{c}$ is usually set a larger value than $p_{m}$.

After the genetic operation, we combine the parents and offspring populations into a whole population of size $2 n_{p}$ and continue the procedures in section IV-B to obtain a new population of size $n_{p}$, which is then used for the genetic operation in section IV-C. The iteration stops when the termination criterion, e.g. a maximum generation gen, is reached.

\section{Deployment Result Update}

Suppose an optimal solution has been obtained using the above NSGA-II algorithm. There is a question that when the coverage thresholds at some PoIs vary, is it required to run the above algorithm once again? As illustrated in Fig.1, the coverage thresholds of PoIs inside the ellipse vary while those outside the ellipse remain the same. One way is to take it as a brand new issue and follow the original steps to reach an optimal solution. Due to the nature of genetic algorithm, this treatment may result in a major change of the node deployment over the whole AoI. In order to preserve the original placement of most sensors, another way is to clip out the varied subarea and run the algorithm independently in the subarea. However, this will bring a problem that nodes outside the subarea cannot contribute to the coverage or connectivity inside, which may result in larger number of nodes than required. Therefore, we propose to do genetic operations in the varied subarea while 


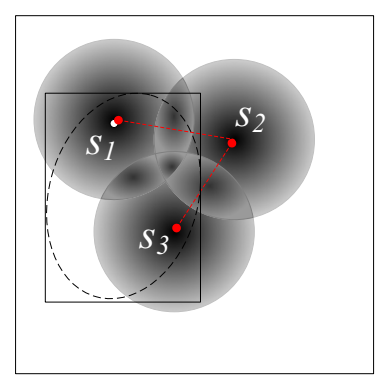

Fig. 1. The coverage requirement in the circled area is varied.

evaluate the objectives still in a global way. This will ensure that nodes outside the varied subarea cooperate with nodes inside in both sensing coverage and communications.

\section{Performance Evaluation}

In this section, we conduct numerical experiments to evaluate the performance of the deployment strategy based on NSGA-II. We compare the algorithm with tabu search (TS) approach proposed in [9], as well as random and grid deployment. In case the coverage threshold in a small area varies after deployment, both global and local operations are conducted to redeploy the sensors. In addition, we compare the efficiency of the two strategies according to multiple metrics.

\section{A. Simulation Settings}

Unless otherwise stated, we use the following settings: (i) $\omega=0.4, \beta=1.2, r_{s}=2 \mathrm{~m}, r_{u}=10 \mathrm{~m}$. (ii) The transmission power increases from $-16.9 \mathrm{~dB}$ to $-3.2 \mathrm{~dB}$, corresponding to equivalent communication ranges from $5 \mathrm{~m}$ to $11 \mathrm{~m}$. The equivalent sensing and communication range under disk models are calculated as below:

$$
\begin{aligned}
& E R_{s}=\int_{0}^{r_{u}} c d h \\
& E R_{c}=\int_{0}^{+\infty} p d h
\end{aligned}
$$

where $c$ is the sensing probability of a sensor within distance $h$ defined in (1), and $p$ is the communication probability defined in (3). Following (11), the equivalent sensing and communication range $\left(P_{t}=-6.9 \mathrm{~dB}\right)$ under disk models equal to $4 \mathrm{~m}$ and $9 \mathrm{~m}$ respectively, as shown in Fig.2. (iii) The deployment region is discretized into $50 \times 50$ grids and the center of each grid is a PoI. The coverage thresholds of the AoI are non-uniformly distributed and vary from 0.1 to 0.99 , as illustrated in the first sub-figure of Fig.8, with light color indicating a large threshold value while dark color on the contrary. (iv) Random deployment approach follows (8) and the best is chosen out of 10 tries. For NSGA-II based strategy, $n_{p}=50, p_{c}=0.75, p_{m}=0.1$, gen $=50$; the settings related to TS strategy can be referred to literature [9]. (v) The connectivity constraint is set to $95 \%$.

\section{B. Simulation Results}

Fig. 3 plots the number of nodes required by four deployment methods under different $E R_{c} / E R_{s}$ ratios. For each
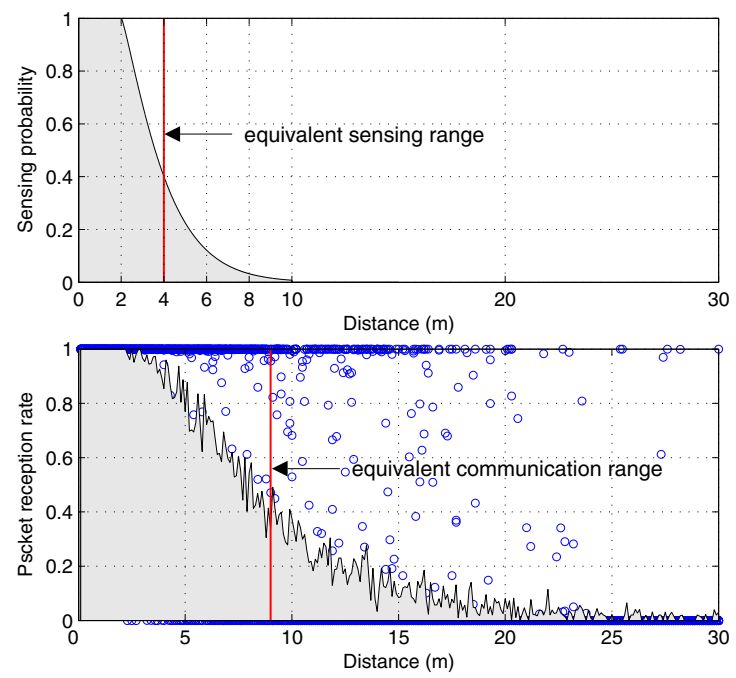

Fig. 2. Sensing and communication models, indicating equivalent sensing and communication range under disk models, with $P_{t}=-6.9 \mathrm{~dB}$.

method, as the $E R_{c} / E R_{s}$ ratio increase from $5 / 4$ to $11 / 4$, the number of nodes required gradually decreases and finally converges to a minimum value. This is because the sensing parameters remain unchanged. There should be a minimum number of nodes guaranteeing the coverage requirement. Further increase in $E R_{s}$ cannot do any help to the coverage but result in a waste of power instead.

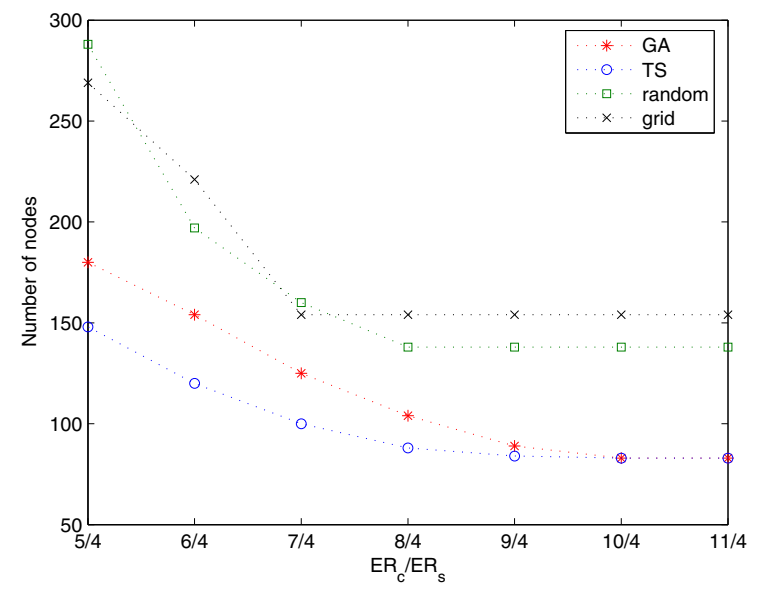

Fig. 3. Required node number of different deployment vs. $E R_{c} / E R_{s}$ ratio.

Although TS based strategy require least sensors, it cannot satisfy the connectivity requirement as shown in Fig.4. This is because TS approach does not support multi-objective optimization problem very well. The strategy proposed in [9] ensures a connected network under boolean model in the initialization phase. However, it does not maintain the connectivity in the afterward searching procedure. The 1connectivity probability is plotted in Fig.4, which indicates that the resulting network by GA approach can ensure a connected network with the probability around $95 \%$.

As $E R_{c} / E R_{s}$ increases, more transmission power is required. As shown in Fig.5, the total power for the network 


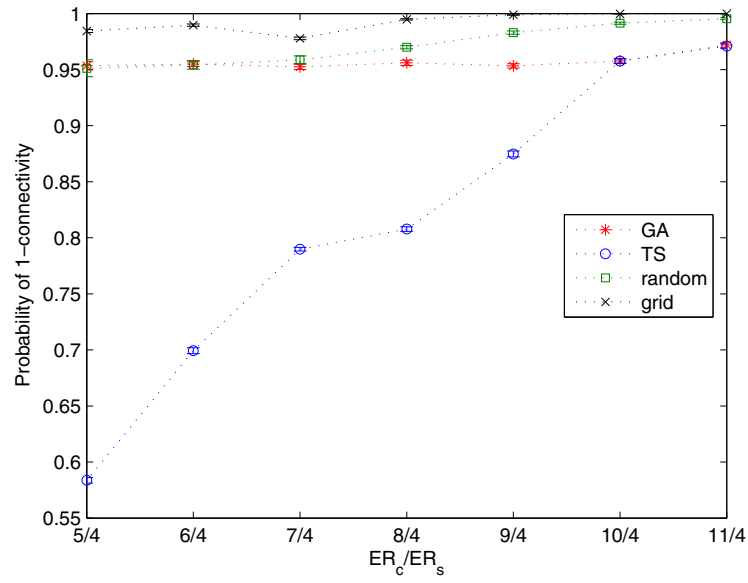

Fig. 4. 1-connectivity probability of differently deployed network vs. $E R_{c} / E R_{s}$ ratio.

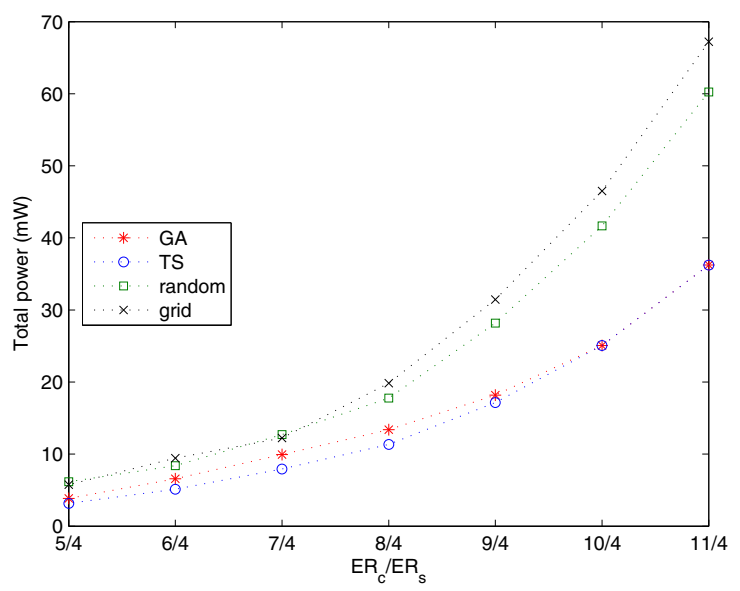

Fig. 5. Total power of different deployment vs. $E R_{c} / E R_{s}$ ratio.

increases in spite of the number of nodes decreasing. It is worth noting that in case $E R_{c} / E R_{s}<2$, the increase in the total power is gentle while in case $E R_{c} / E R_{s}>2$, the increase becomes steep. This implies that there should be a tradeoff between transmission power setting and the total number of nodes required, preventing the total power from going to the steeply increase range.

Fig.6 and Fig. 7 show the obtained nondominated solutions using NSGA-II. In Fig.6, all the solutions satisfy the constraint $C t$, nondominated solutions with tradeoffs in $F_{1}$ and $F_{2}$ are presented. Similarly in Fig.7, all the solutions satisfy $F_{2}=$ 0 , nondominated solutions with tradeoffs in $F_{1}$ and $C t$ are given for users to choose an appropriate one according to their practical conditions and limits.

In case that the coverage requirement in some subarea vary, we do both global and local optimizations for the updated problem. As shown in Fig.8, the first sub-figure denotes the original requirement, while the 4 sub-figures afterwards are new requirement with subareas variation. Fig.9 shows respective performance. We can see that the total number of nodes

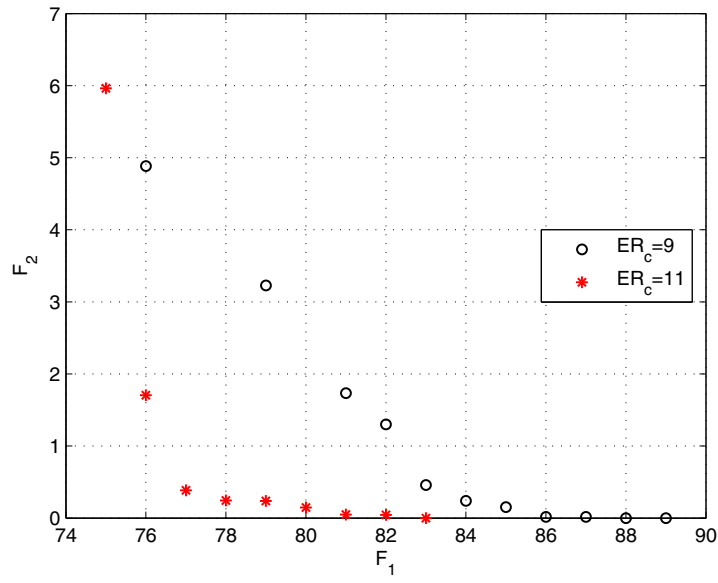

Fig. 6. Nondominated solutions with $E R_{c}=9$ and $E R_{c}=11, C t \geq 95 \%$.

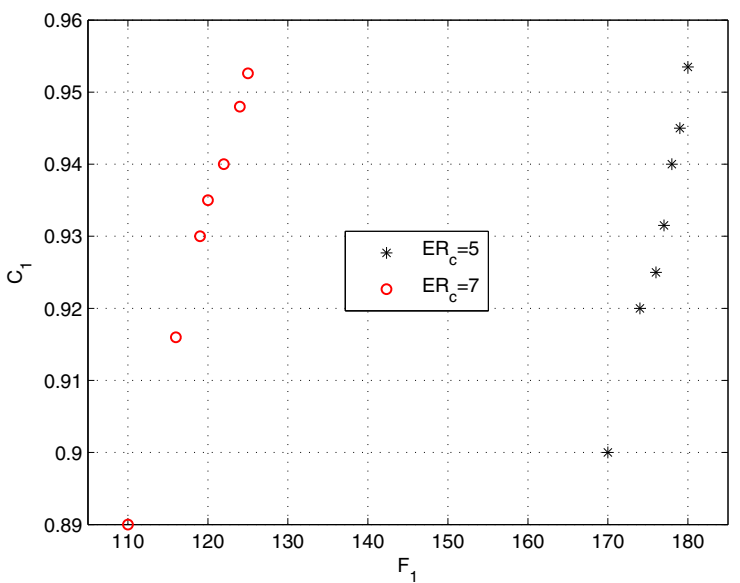

Fig. 7. Nondominated solutions with $E R_{c}=5$ and $E R_{c}=7, F_{2}=0$.

required is almost the same for the two approaches. Local approach converges more quickly and it is easier to find a novel solution while the global approach which searches in a wider range, lacks of efficiency and sometimes may not reach the same level as local approach with the same time complexity. An obvious advantage of local approach lies in the varied number of nodes in the network. For global genetic operation, it is highly possible that the deployment in the area without threshold variation be destroyed to satisfy a global search of optimal solution. On the contrary, local approach restricts the search in the localized area. Thus the placement outside is not affected. Since the fitness function is still evaluated in a global way, the local approach will not degrade too much, if compared with the optimal solutions. Both local and global approaches satisfy the connectivity constraints as shown in Fig.10.

\section{Complexity Analysis}

The worst-case complexity of NSGA-II is $O\left(m n_{p}^{2}\right)$, where $m$ is the number of objectives while for TS it is $O(k X Y)$, where $k$ is a constant related to iteration time and sensing 

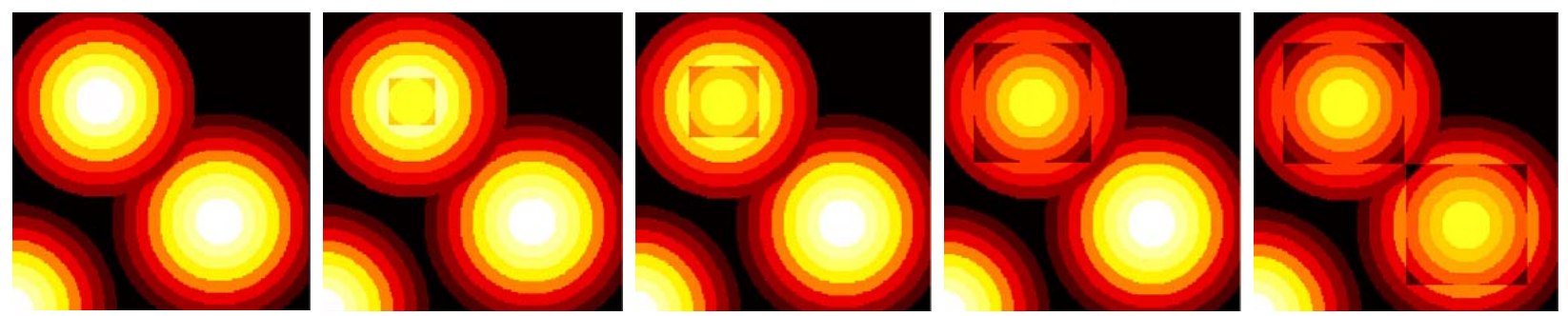

Fig. 8. The coverage requirement in subareas is varied: four situations.

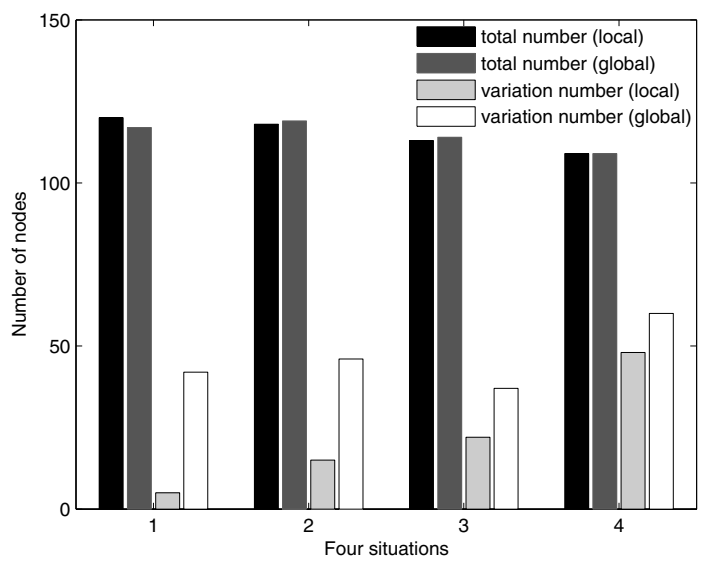

Fig. 9. Total and varied number of nodes by local and global computations

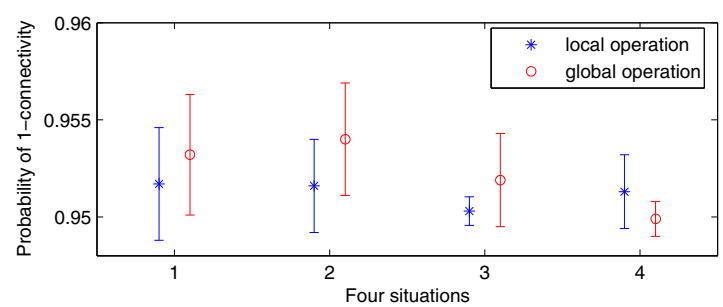

Fig. 10. 1-connectivity probability by local and global computations

range. It is worth noting that if the deployment area is large or the grid granularity is small, the complexity of NSGAII based strategy will not be affected but the complexity of TS based method will increase. In this sense, NSGA-II based deployment strategy is more adaptive and robust to the dimension and granularity of the area.

\section{CONCLUSions}

We propose a novel NSGA-II based deployment strategy, which is able to meet the desired coverage performance and maintain connectivity in a probabilistic manner with a relatively small number of sensors. Besides, for the applications in which the coverage requirement varies in some subareas, a local genetic operation is more time-efficient and requires less variation in the original disposal than a renewed global optimization. Our solution to the deployment issue can also be applied to solve on-duty sensor set selection and scheduling problem if the sensors have already been densely deployed. A design of hybrid algorithm is worked in progress to find the best genetic operator and improve the searching efficiency.

\section{REFERENCES}

[1] H. Ammari and S. Das, "Integrated coverage and connectivity in wireless sensor networks: A two-dimensional percolation problem," IEEE Trans. Comput., vol. 57, no. 10, pp. 1423-1434, 2008.

[2] X. Wang, G. Xing, Y. Zhang, C. Lu, R. Pless, and C. Gill, "Integrated coverage and connectivity configuration in wireless sensor networks," in ACM SenSys, 2003, pp. 2839.

[3] X. Bai, D. Xuan, Z. Yun, T. Lai, and W. Jia, "Complete optimal deployment patterns for full-coverage and $k$ connectivity $(k \leq 6)$ wireless sensor networks," in ACM MobiHoc, 2008, pp. 401-410.

[4] K. Deb, A. Pratap, S. Agarwal, and T. Meyarivan, "A fast and elitist multiobjective genetic algorithm: NSGA-II," IEEE Trans. Evol. Comput., vol. 6, no. 2, pp. 182-197, 2002.

[5] R. K. amd H. Liu and H.-H. Chen, "Reduced complexity intrusion detection in sensor networks using genetic algorithm," in IEEE ICC, 2009.

[6] M. Alabau, L. Idoumghar, and R. Schott, "New hybrid genetic algorithms for the frequency assignment problem," IEEE Trans. Broadcast., vol. 48, no. 1, pp. 27-34, 2002.

[7] Y. Zou and K. Chakrabarty, "Uncertainty-aware and coverage-oriented deployment for sensor networks," $J$. Parallel and Distrib. Comput., vol. 64, no. 7, pp. 788798, 2004.

[8] J. Zhang, T. Yan, and S. Son, "Deployment strategies for differentiated detection in wireless sensor networks," in IEEE SECON, 2006, pp. 316-325.

[9] N. Aitsaadi, N. Achir, K. Boussetta, and G. Pujolle, "Heuristic Deployment to Achieve Both Differentiated Detection and Connectivity in WSN," in IEEE VTC Spring, 2008, pp. 123-127.

[10] G. Tan, S. Jarvis, and A. Kermarrec, "Connectivityguaranteed and obstacle-adaptive deployment schemes for mobile sensor networks," in IEEE ICDCS, 2008, pp. 429-437.

[11] M. Zuniga and B. Krishnamachari, "Analyzing the transitional region in low power wireless links," in IEEE SECON, 2004, pp. 517-526.

[12] Crossbow Motes. http://www.xbow.com. 